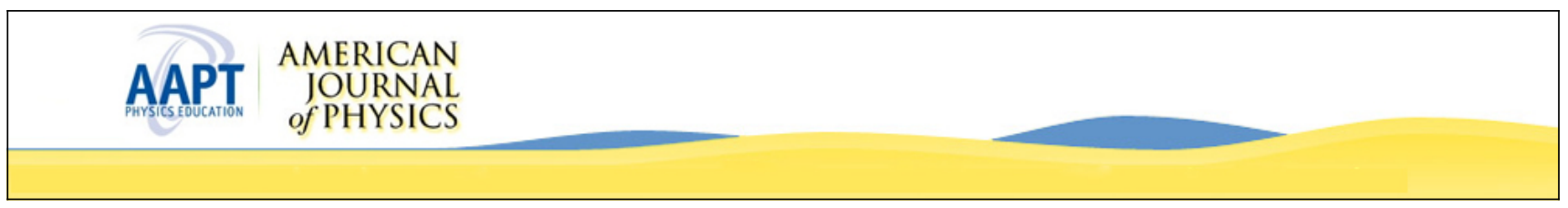

\title{
Comparison of electromagnetic and gravitational radiation: What we can learn about each from the other
}

Richard H. Price, John W. Belcher, and David A. Nichols

Citation: Am. J. Phys. 81, 575 (2013); doi: 10.1119/1.4807853

View online: http://dx.doi.org/10.1119/1.4807853

View Table of Contents: http://ajp.aapt.org/resource/1/AJPIAS/v81/i8

Published by the American Association of Physics Teachers

\section{Related Articles}

Resource Letter: GrW-1: Gravitational waves

Am. J. Phys. 71, 520 (2003)

Answer to Question \#49. Why c for gravitational waves?

Am. J. Phys. 65, 590 (1997)

Answer to Question \#49. Why c for gravitational waves?

Am. J. Phys. 65, 591 (1997)

Mechanical amplifiers for gravitational wave detectors

Am. J. Phys. 65, 574 (1997)

If light waves are stretched by gravitational waves, how can we use light as a ruler to detect gravitational waves? Am. J. Phys. 65, 501 (1997)

\section{Additional information on Am. J. Phys.}

Journal Homepage: http://ajp.aapt.org/

Journal Information: http://ajp.aapt.org/about/about_the_journal

Top downloads: http://ajp.aapt.org/most_downloaded

Information for Authors: http://ajp.dickinson.edu/Contributors/contGenlnfo.html

\section{ADVERTISEMENT}

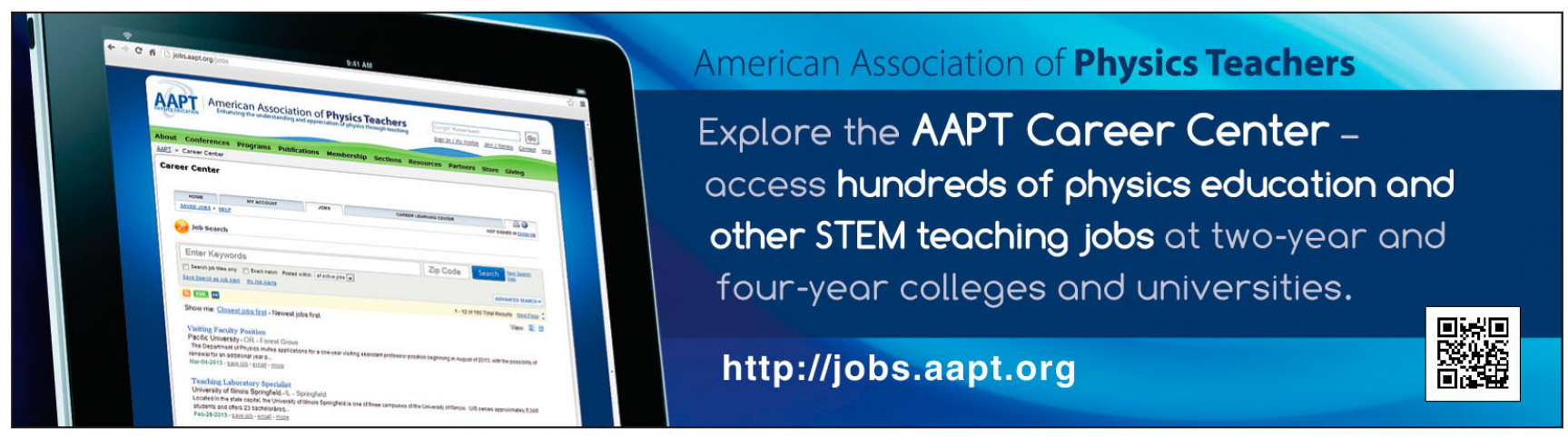




\title{
Comparison of electromagnetic and gravitational radiation: What we can learn about each from the other
}

\author{
Richard H. Price ${ }^{\text {a) }}$ \\ Department of Physics and Astronomy, University of Texas at Brownsville, Brownsville, Texas 78520 \\ John W. Belcher ${ }^{\text {b) }}$ \\ Department of Physics, Massachusetts Institute of Technology, Cambridge, Massachusetts 02139 \\ David A. Nichols ${ }^{\mathrm{c})}$ \\ Theoretical Astrophysics, California Institute of Technology, Pasadena, California 91125
}

(Received 18 December 2012; accepted 13 May 2013)

\begin{abstract}
We compare the nature of electromagnetic fields and gravitational fields in linearized general relativity. We carry out this comparison both mathematically and visually. In particular, the "lines of force" visualizations of electromagnetism are contrasted with the recently introduced tendex/vortex eigenline technique for visualizing gravitational fields. Specific solutions, visualizations, and comparisons are given for an oscillating point quadrupole source. Among the similarities illustrated are the quasistatic nature of the near fields, the transverse $1 / r$ nature of the far fields, and the interesting intermediate field structures connecting these two limiting forms. Among the differences illustrated are the meaning of field line motion and of the flow of energy. (c) 2013 American Association of Physics Teachers.

[http://dx.doi.org/10.1119/1.4807853]
\end{abstract}

\section{INTRODUCTION}

There are many similarities between electromagnetic (EM) radiation and gravitational radiation: both travel at the speed of light; both carry energy away from their sources; both consist of transverse waves with two polarizations. In addition, Einstein's general relativity, the theoretical underpinning of gravitational waves, can be put into a form remarkably parallel to Maxwell's electrodynamics, the theoretical underpinning of EM waves. Despite the many similarities, there are important differences and focusing on those differences helps to give a deeper understanding of both kinds of radiation.

Our mechanism for exploring these differences will be the visualization of the fields. Visualization of EM field lines has proven very helpful to student understanding. ${ }^{1}$ The visualization of gravitational fields has been a challenge, but the recently developed technique of using "tendex" and "vortex" lines $^{2-4}$ provides insights that may be of pedagogical value comparable to the plotting of electric or magnetic field lines in EM.

Both in EM and in gravitation, the dynamic nature of radiation fields is of central importance to visualization, and in both EM and gravitation, waves carry energy. In EM, it will turn out that a definite meaning can be given to the motion of field lines and to the transport of energy. By contrast, in the gravitational case, a definite meaning cannot be given to either of these concepts. This contrast will help us understand some important ways in which gravitation fundamentally differs from EM.

The rest of this paper is organized as follows. In Sec. II A, we give a brief review of EM theory to serve as a basis for comparison with the elements of gravitational theory that we subsequently present in Sec. II B. Section III develops the principles of visualization of static fields, for EM in Sec. III A, and for gravitation in Sec. III B. In Sec. III, to illustrate both EM and gravitational static fields, we focus on a particular model that will be useful later in the discussion of radiation. A dipole is the simplest model for a source of EM radiation, but a gravitational dipole cannot generate radiation. We therefore choose the simplest configuration that can generate both EM and gravitational waves: a point quadrupole.

For simplicity of visualization, we eliminate the issues of visualizing truly three dimensional fields by having the point quadrupole be axisymmetric. Time-changing sources give rise to radiation fields for both EM and gravitation. These dynamical fields and their visualization are discussed in Sec. IV with the example of oscillating EM and gravitational quadrupoles. We summarize and restate our conclusions in Sec. V.

A few words are in order about our choices for notation and conventions. Our goal has been to produce a paper that can be understood by a reader with a minimum of mathematical preliminaries. To that end we have avoided certain practices that are common in advanced literature. The following points deserve particular notice. (i) Papers involving relativity typically assume units in which $c$, the speed of light, is taken to be unity. In order to have expressions in which the dimensionality of quantities is more transparent, we do not make that choice; all factors of $c$ explicitly appear. (ii) It is common to use the "Einstein summation convention," in which summation is assumed for any repeated index. Adopting this convention would allow us to drop the explicit summation symbols in Eq. (3) and many subsequent equations. We have chosen, however, to show these summation symbols explicitly. (iii) To avoid the mathematical baggage of covariant differentiation, we have been explicit in using only Cartesian coordinates and Cartesian components where expressions involve differentiation, as in Eq. (4). (iv) We avoid "coordinate bases" that are commonly used in computations with tensor fields. Rather, the components expressed, e.g., in Eq. (18), are with respect to the familiar spherical coordinate orthonormal basis, not the coordinate basis. One of the simplifications following from this choice is that indices on components are the same whether they are superscripts or subscripts; their location is chosen for 
convenience. (v) We use rationalized MKS units throughout for electromagnetism, since this is the system that is familiar to most students.

\section{INTRODUCTORY THEORY}

\section{A. Electromagnetic fields}

For comparison with the gravitational case, it is useful to recall the very roots of electromagnetic physics. The electric field $\mathbf{E}$ and magnetic field $\mathbf{B}$ are defined through the expression for the acceleration a due to the total electromagnetic force, the Lorentz force, acting on a point particle of mass $m$ and charge $q$, moving at velocity $\mathbf{v}^{5}$

$$
\mathbf{a}=\frac{q}{m}(\mathbf{E}+\mathbf{v} \times \mathbf{B}) .
$$

The fields obey the Maxwell equations, which in rationalized MKS units take the form

$$
\begin{aligned}
& \nabla \cdot \mathbf{E}=0, \quad \nabla \times \mathbf{E}+\frac{\partial \mathbf{B}}{\partial t}=0, \\
& \nabla \times \mathbf{B}-\frac{1}{c^{2}} \frac{\partial \mathbf{E}}{\partial t}=0, \quad \nabla \cdot \mathbf{B}=0 .
\end{aligned}
$$

Here, we have simplified the equations by assuming a region devoid of sources and of material properties - we take the charge and current density to be zero and assume vacuum values of the dielectric constant and magnetic permeability. It will be useful to rewrite the Lorentz and Maxwell equations in component form (in which we assume a Cartesian basis)

$$
\begin{aligned}
& a^{j}=\frac{q}{m}\left(E^{j}+\sum_{k, p} \epsilon_{j k p} v^{k} B^{p}\right), \\
& \sum_{j} \frac{\partial E_{j}}{\partial x^{j}}=0, \quad \sum_{j, k} \epsilon_{i j k} \frac{\partial E_{k}}{\partial x^{j}}+\frac{\partial B_{i}}{\partial t}=0, \\
& \sum_{j, k} \epsilon_{i j k} \frac{\partial B_{k}}{\partial x^{j}}-\frac{1}{c^{2}} \frac{\partial E_{i}}{\partial t}=0, \quad \sum_{j} \frac{\partial B_{j}}{\partial x^{j}}=0 .
\end{aligned}
$$

Here, the summations are over the indices of the Cartesian components and coordinates, $\left\{x^{1}, x^{2}, x^{3}\right\}$ or $\{x, y, z\}$. The symbol $\epsilon_{i j k}$ is the three-dimensional alternating symbol used in the construction of determinants and cross products. It vanishes if any of its indices is repeated (e.g., $\epsilon_{221}=0$ ), equals +1 for any even permutation of 123 or $x y z$ $\left(\epsilon_{123}=\epsilon_{312}=\epsilon_{231}=1\right)$, and equals -1 for any odd permutation $\left(\epsilon_{213}=\epsilon_{321}=\epsilon_{132}=-1\right)$.

The simplest solutions of the Maxwell equations are the time independent solutions, especially the point multipole (point charge, dipole, quadrupole, ...) solutions for electrostatic or magnetostatic fields. Of greatest interest in this paper will be the not-so-simple radiation solutions in which time variation is essential. Of considerable importance to these radiation solutions is the Poynting vector, the flux of electromagnetic power per unit cross sectional area, given by

$$
\mathbf{P}=\frac{1}{\mu_{0}} \mathbf{E} \times \mathbf{B} .
$$

In a general treatment, the computation of the radiation produced by a given distribution of time-changing charges and currents leads to retarded integrals over those sources. Here, however, we are primarily interested in the description and visualization of the resulting fields, so we simply invoke radiation fields without being specific about the internal details of their source. For such purposes, the choice usually made is that of a point dipole, but for our purpose, this choice has the disadvantage that it has no gravitational ana$\log$. The lowest-order multipole for gravitational radiation is the quadrupole. ${ }^{6}$ Accordingly, as our example of an electromagnetic radiating source we choose a point quadrupole, and we make the description and visualization as simple as possible by taking the quadrupole to be axisymmetric.

\section{B. Gravitational fields}

To understand what is meant by "gravitation" in relativistic theories it is best to start with the simplest case: gravitostatics (i.e., gravitation for static configurations). In this case, if the gravitational fields are typically weak-if they do not drive particles to speeds comparable to $c$ - then Newtonian ideas can be adopted with minor modification to relativistic gravitation. By "gravitational field" in this approach, we do not mean, e.g., the downward acceleration of $9.8 \mathrm{~m} / \mathrm{s}^{2}$ near the surface of the Earth. More generally, if $\Phi_{g}$ is the usual Newtonian potential, then $\nabla \Phi_{g}$ is not considered "true" gravitation. Because gravitation affects all particles identically its effects on particles undergoing their natural, freely falling motion disappear in a freely falling frame, the inertial frame in the relativistic view of spacetime.

Gravitation, in the relativistic viewpoint, is the way in which the natural free-fall motions vary from place to place and from time to time. In a static configuration-one in which there is no change from time to time-the information is contained in the way in which $\nabla \Phi_{g}$ varies from place to place. The rate of variation of a vector is a tensor; in the case of $\nabla \Phi_{g}$ it is often called the gravitoelectric field. ${ }^{7}$ (Because this tensor describes the raising of tides on astrophysical objects in a Newtonian setting it is also called the tidal tensor.) In a Cartesian basis, the content of this tensor is the set of tensor components 8

$$
\mathcal{E}_{j k}=\frac{\partial^{2} \Phi_{g}}{\partial x^{j} \partial x^{k}} .
$$

The trace of this gravitoelectric tensor is a familiar quantity

$$
\sum_{k} \mathcal{E}_{k k}=\sum_{k} \frac{\partial^{2} \Phi_{g}}{\partial x^{k} \partial x^{k}}=\nabla^{2} \Phi_{g} .
$$

Just as the electric field is divergenceless outside sources, the gravitoelectric field is traceless outside sources.

The definition in Eq. (7) is valid even in the presence of sources. In this case, the right hand side has the familiar value $4 \pi G$ times mass density. This equation, then, is analogous to Coulomb's law for electromagnetism. It gives a definition of the field in terms of its sources, but only for a static field. To deal with radiation, we need more general definitions, definitions based on the manifestations - the physical effects - of these fields. In the case of electromagnetism, this more general definition is given by Eq. (1) or, equivalently, Eq. (3). In relativistic gravitation, there is no concept of force per se. Rather, the manifestations of gravity are seen in the effects on two point particles separated by a small 
displacement $\mathbf{s}$ and with a relative velocity $\mathbf{v}$. The relative acceleration of the two particles, given by ${ }^{9}$

$$
\frac{d^{2} s^{j}}{d t^{2}}=-\sum_{k} \mathcal{E}_{j k} s^{k}-2 \sum_{k, p, m} \epsilon_{j k p} \mathcal{B}_{p m} v^{k} s^{m},
$$

defines the tensors $\mathcal{E}$ and $\mathcal{B}$. The similarity to the Lorentz acceleration in Eq. (3) is striking, especially if one considers $\mathcal{E}_{j k} s^{k}$ and $\mathcal{B}_{j k} s^{k}$ to be vectors. There are, of course, differences of detail, one of which is very fundamental. In Eq. (1) or (3) the factor $q / m$ describes the special features of the particle undergoing electromagnetic acceleration. By contrast, in Eq. (8), there is no reference to any characteristic of the particles. In accordance with the so-called "equivalence principle," gravitation acts in the same way on any particle.

The gravitoelectric and gravitomagnetic fields defined by Eq. (8) are symmetric $\left(\mathcal{E}_{j k}=\mathcal{E}_{k j}, \mathcal{B}_{j k}=\mathcal{B}_{k j}\right)$. Outside sources, these fields are traceless $\left(\sum_{k} \mathcal{E}_{k k}=\sum_{k} \mathcal{B}_{k k}=0\right)$ and obey the Maxwell-like relations ${ }^{10}$

$$
\begin{aligned}
& \sum_{j} \frac{\partial \mathcal{E}_{j k}}{\partial x^{j}}=0, \\
& \frac{1}{2}\left(\sum_{j k} \epsilon_{p j k} \frac{\partial \mathcal{E}_{q k}}{\partial x^{j}}+\sum_{j k} \epsilon_{q j k} \frac{\partial \mathcal{E}_{p k}}{\partial x^{j}}\right)+\frac{\partial \mathcal{B}_{p q}}{\partial t}=0, \\
& \frac{1}{2}\left(\sum_{j k} \epsilon_{p j k} \frac{\partial \mathcal{B}_{q k}}{\partial x^{j}}+\sum_{j k} \epsilon_{q j k} \frac{\partial \mathcal{B}_{p k}}{\partial x^{j}}\right)-\frac{1}{c^{2}} \frac{\partial \mathcal{E}_{p q}}{\partial t}=0, \\
& \sum_{j} \frac{\partial \mathcal{B}_{j k}}{\partial x^{j}}=0,
\end{aligned}
$$

which can be written as

$$
\begin{aligned}
& \nabla \cdot \mathcal{E}=0, \quad \nabla \times \mathcal{E}+\frac{\partial \mathcal{B}}{\partial t}=0, \\
& \nabla \times \mathcal{B}-\frac{1}{c^{2}} \frac{\partial \mathcal{E}}{\partial t}=0, \quad \boldsymbol{\nabla} \cdot \mathcal{B}=0,
\end{aligned}
$$

with appropriate interpretation of the divergence and curl. Note that the divergence can be taken on either index (since the "gravito-" tensors are symmetric) and the curl in these equations is symmetrized. ${ }^{11}$

Two theoretical points bear mentioning: (i) Just as the six independent components of $\mathbf{E}$ and $\mathbf{B}$ contain a complete description of the electromagnetic field at a point, the ten independent components of the two symmetric traceless tensors $\mathcal{E}$ and $\mathcal{B}$ contain a complete description of the gravitational field at a point. (ii) The vectors $\mathbf{E}$ and $\mathbf{B}$ are "gauge invariant." They cannot, for instance, be made to vanish by a mathematical choice. In the same sense, $\mathcal{E}$ and $\mathcal{B}$ are gauge invariant. ${ }^{12}$ This is the mathematical equivalent of the physical statement that these quantities are directly physically measurable.

\section{STATIC FIELDS}

\section{A. Electromagnetism}

\section{General considerations for electromagnetic visualization}

The electric and magnetic fields are vector fields, and hence in principle are simple to picture. One can use small arrows indicating field direction, with arrow length indicating vector magnitude. In practice, this method is usually awkward; instead one abandons the need to display vector magnitudes and links the vector directions together into "field lines," curves whose direction at any point indicates the direction, at that point, of the vector field. A plot of these field lines throughout a region of interest gives a good intuitive impression of the nature of the vector field. In addition, one can (but rarely does) draw the plot of field lines so that the density of lines in the neighborhood of a point is proportional to the magnitude of the vector field at that point. This is a consistent procedure for a divergenceless vector field, like the electric field in a source-free region.

Whether small arrows or field lines are used, the limitations of spatial resolution are often a serious barrier to useful visualization. Below we will be illustrating vector fields, and later eigenline fields, with a newer technique: the line integral convolution (LIC) method of Cabral and Leedom. ${ }^{13}$ In this method, the brightness or darkness of pixels is correlated along field lines. The method produces images with streaks showing the structure of the field lines in an intuitively appealing way and with resolution approaching that of the display.

\section{Static electromagnetic point quadrupole}

As the simplest example of an electromagnetic multipole source that will be generalizable to gravitation, we choose an axisymmetric quadrupole. A realization of such a source is shown in Fig. 1: two equal positive charges symmetrically arranged on the $z$-axis about a double negative charge at the origin. Because there is no net charge and no favored positive direction, the configuration has neither a monopole nor a dipole moment. The static configuration pictured then has a quadrupole as its lowest nonvanishing multipole.

In general, the Cartesian components of an electric quadrupole are given by ${ }^{14}$

$$
Q_{i j}=\int\left(3 x_{i} x_{j}-r^{2} \delta_{i j}\right) \rho(\mathbf{x}) d^{3} x,
$$

where $\rho(\mathbf{x})$ is charge density. For our model in Fig. 1, the specific components are

$$
Q_{z z}=4 d^{2} q \equiv 4 Q, \quad Q_{x x}=Q_{y y}=-2 Q,
$$

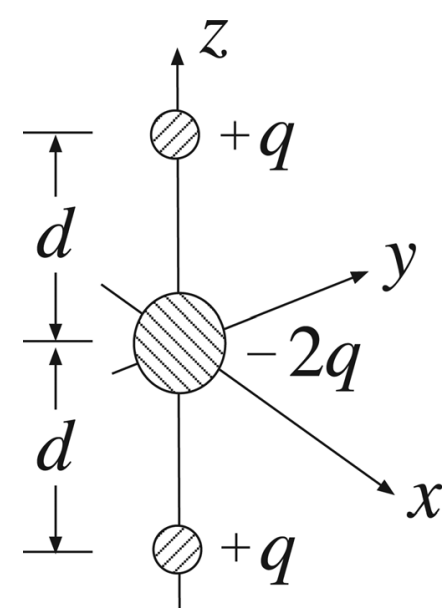

Fig. 1. A simple model of an electric quadrupole. 
with $Q_{i j}=0$ for $i \neq j$. Note that the quadrupole $Q_{i j}$ is itself a tensor describing the charge distribution of the source. It is not a tensor field. The electric field produced by that source is the vector field $\mathbf{E}$.

The model in Fig. 1 is not a "pure" quadrupole; it has multipole moments of order 2, 4, 6... More importantly, it is not a "point source"; it has a characteristic size $d$. To create a pure point quadrupole we use a limiting process analogous to that for defining a point dipole. We shrink $d$ to zero, while keeping finite the product $Q \equiv d^{2} q$. The result is a point source with only a quadrupole moment.

The static electric field for the point quadrupole is most simply computed from the electrostatic potential. A formal procedure $^{14}$ can be used to find the potential directly from the quadrupole components in Eq. (12), or a limiting procedure can be applied to the potential of the three point charges in Fig. 1. The resulting electrostatic potential is

$$
\Phi_{e}=\frac{Q}{4 \pi \epsilon_{0} r^{3}}\left(3 \cos ^{2} \theta-1\right)
$$

and hence the electric field $\mathbf{E}=-\nabla \Phi_{e}$ has spherical components

$$
E_{r}=\frac{6 Q}{4 \pi \epsilon_{0} r^{4}}\left(\frac{3}{2} \cos ^{2} \theta-\frac{1}{2}\right), \quad E_{\theta}=\frac{6 Q}{4 \pi \epsilon_{0} r^{4}} \cos \theta \sin \theta .
$$

The electric field topology for this case is illustrated in Fig. 2; for this static electric configuration there are no associated magnetic fields.

\section{B. Gravitation}

\section{General considerations for gravitational visualization}

The problem of visualizing the $\mathcal{E}_{j k}$ and $\boldsymbol{B}_{j k}$ fields is a special example of the question: how does one visualize tensor fields? The gravito- fields $\mathcal{E}_{j k}$ and $\mathcal{B}_{j k}$ are tensors, but at least they are the simplest nontrivial type of three-dimensional tensor: they are second rank (two index) symmetric tensors. In this sense, they are similar to the most familiar tensors of physics such as the inertia tensor, the stress tensor, the dielectric tensor of an anisotropic material, etc.

In the case of a tensor like the inertia tensor of an extended massive object, a reasonable visualization is the inertia

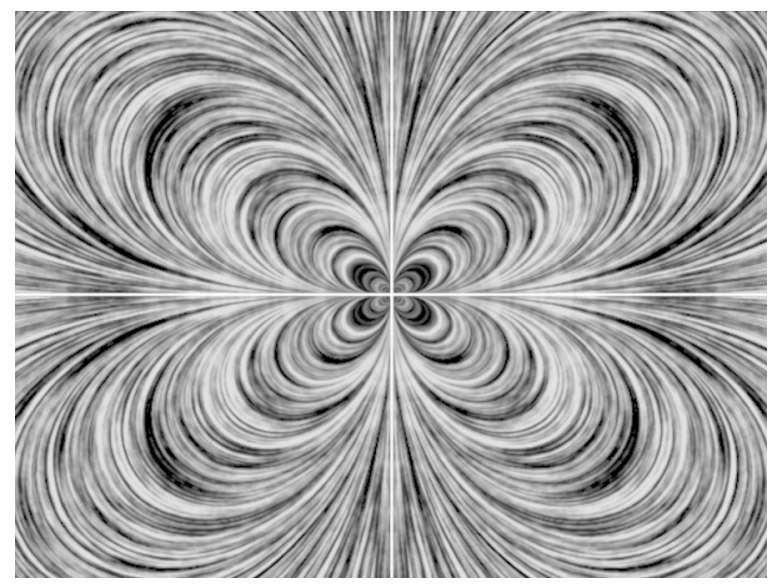

Fig. 2. A line integral convolution of the electric field lines of the azimuthally symmetric static electric quadrupole described in the text. ellipsoid: a three dimensional ellipsoid whose shape shows the directions and size of the principal axes of the moment of inertia of the massive object. ${ }^{15}$ This ellipsoid for a secondrank symmetric tensor is very much the analog of the arrow for a vector.

The moment of inertia ellipsoid describes a single tensor, not a tensor field. While a display of space filled with arrows has some usefulness for the visualization of a vector field, the same is probably not true of space filled with ellipsoids. But, just as arrows can be connected together to form field lines, the principal axes of tensorial ellipsoids can be connected to form a network of lines with visualization properties somewhat similar to field lines. The specific technique for visualization of a symmetric tensor $A_{j k}$ is to find, at each point in space, the principal directions- the vectors $v^{k}$ satisfying the eigenvector condition $A_{j k} v^{k}=\lambda v^{k}$. If $A_{j k}$ is symmetric, we are guaranteed that at any point three such eigenvectors exist and are orthogonal. Just as the directions of vectors are connected to form a field line, we can connect together the directions of eigenvectors to form an "eigenline." At any point the tangent to an eigenline is parallel to one of the eigenvectors of the tensor.

A simple example of this is provided by the spherically symmetric gravitational field of a body of mass $M$

$$
\Phi_{g}=-\frac{G M}{\sqrt{x^{2}+y^{2}+z^{2}}} .
$$

The components of the gravitoelectric field can be computed in Cartesian coordinates using Eq. (7), but the results are most simply expressed using the spherical basis. The only nonvanishing components are

$$
\mathcal{E}_{r r}=-\frac{2 G M}{r^{3}}, \quad \mathcal{E}_{\theta \theta}=\frac{G M}{r^{3}}, \quad \mathcal{E}_{\phi \phi}=\frac{G M}{r^{3}} .
$$

The eigenvectors of the corresponding diagonal matrix are clear: there is an eigenvector in the radial direction and a degenerate pair of eigenvectors in the $\theta$ and $\phi$ directions. One family of eigenlines, then, is the set of radial lines, but there is some ambiguity in the other two families; any curve lying in a surface of constant radius will be an eigenline. This ambiguity is an expected consequence of the degeneracy of the nonradial eigenvectors. A convenient choice for the nonradial eigenlines is the coordinate lines of $\theta$ and $\phi$ in a spherical coordinate system.

Eigenlines have been used for some time in the visualization of stress (a tensor quantity) in fluid dynamics and solid mechanics ${ }^{16}$ and have recently been suggested for use in visualizing the gravitoelectric and gravitomagnetic fields. ${ }^{2-4}$ Those advocating the application to gravitation give the name vortex lines to the eigenvectors of $\mathcal{B}$ due to the role of that field in driving the precession of spin. The eigenvector field lines of $\mathcal{E}$ are given the name tendex lines, suggestive of the role of the tidal distortions associated with $\mathcal{E}$. This approach to visualization holds the promise of giving the kind of intuitive insights that may suggest what configurations lead to strong emission of power and of linear momentum in gravitational waves.

\section{Static gravitational point quadrupole}

We now consider the gravitational equivalent of the configuration in Fig. 1. Here, the objects at $z= \pm d$ are points of 
mass $M$, rather than points of charge $q$. In analogy with Fig. 1, we include a negative mass $-2 M$ at the center. ${ }^{17}$ The quadrupole components for the gravitational case are given by the same integral as that in Eq. (11), but with $\rho$ now representing mass density. The components therefore are those in Eq. (12) with $q$ replaced by $M$.

The Newtonian gravitational potential for the mass configuration in Fig. 3 is the same as the electrostatic potential of the charge configuration in Fig. 1 after the replacements $q \rightarrow M$ and $1 / 4 \pi \epsilon_{0} \rightarrow-G$. The limit of this potential, for $d \rightarrow 0$ with $Q=M d^{2}$ fixed, is

$$
\Phi_{g}=-\frac{G Q}{r^{3}}\left(3 \cos ^{2} \theta-1\right) .
$$

The components of the static gravitoelectric field are straightforward to compute from this potential. ${ }^{18}$ For the static gravitational quadrupole, in analogy with the electric case, there are no gravitomagnetic fields. ${ }^{19}$ The components of the gravitoelectric fields, in a spherical basis, are given by

$$
\begin{aligned}
& \mathcal{E}_{r r}=-\frac{12 G Q}{r^{5}}\left(3 \cos ^{2} \theta-1\right), \\
& \mathcal{E}_{r \theta}=-\frac{24 G Q}{r^{5}} \sin \theta \cos \theta, \\
& \mathcal{E}_{\phi \phi}-\mathcal{E}_{\theta \theta}=\frac{6 G Q}{r^{5}} \sin ^{2} \theta .
\end{aligned}
$$

The components $\mathcal{E}_{r \phi}$ and $\mathcal{E}_{\theta \phi}$ are zero by axisymmetry, and the individual components $\mathcal{E}_{\phi \phi}$ and $\mathcal{E}_{\theta \theta}$ follow from the last of Eqs. (18) and from the tracelessness condition $\mathcal{E}_{\phi \phi}+\mathcal{E}_{\theta \theta}=-\mathcal{E}_{r r}$.

We now turn to the issue of visualizing these fields. The structure of the components of $\mathcal{E}$ shows that two of the eigenvectors will be in the $r \theta$-plane, and one in the $\phi$-direction. The eigenlines in the $\phi$-direction are simple azimuthal circles. The much more interesting eigenlines in the $r \theta$-plane are shown, as LIC images, in Fig. 4.

The comparison of Figs. 4 and 2 is very instructive and poses a sequence of questions. The electrostatic and gravitostatic potentials are identical aside from trivial replacements. Why are the visualizations so different? The immediate answer is that in the electromagnetic case we are picturing $\mathbf{E}=-\nabla \Phi_{e}$, a vectorial quantity with components $\partial \Phi_{e} / \partial x^{j}$. In the gravitational case, we are picturing not the vector field

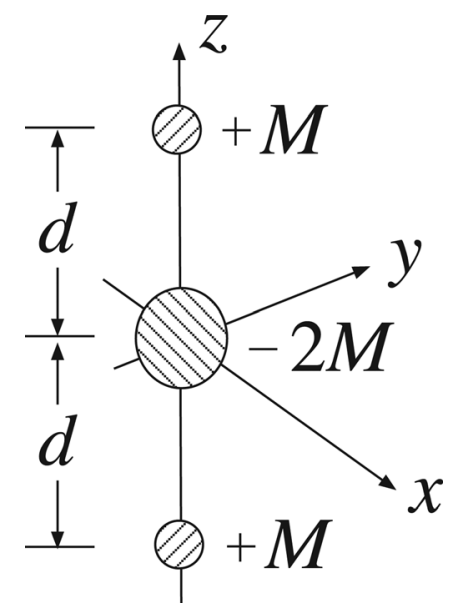

Fig. 3. A simple model of a gravitational quadrupole.

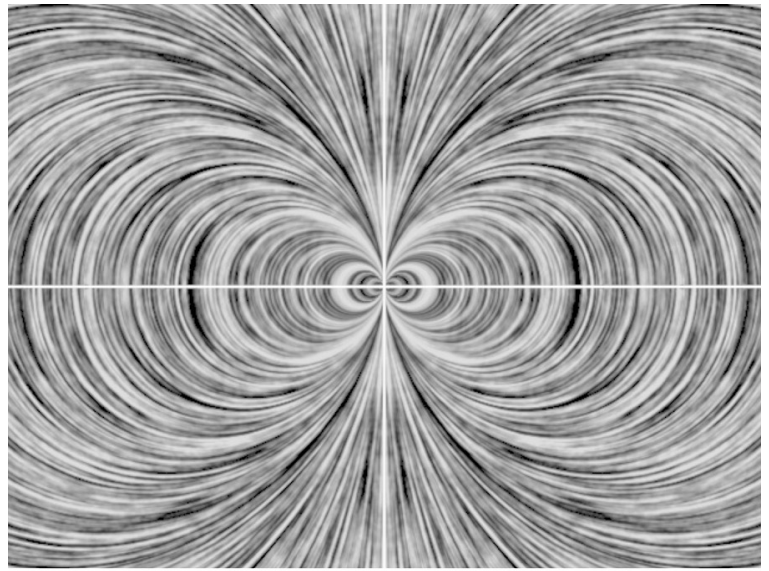

(a)

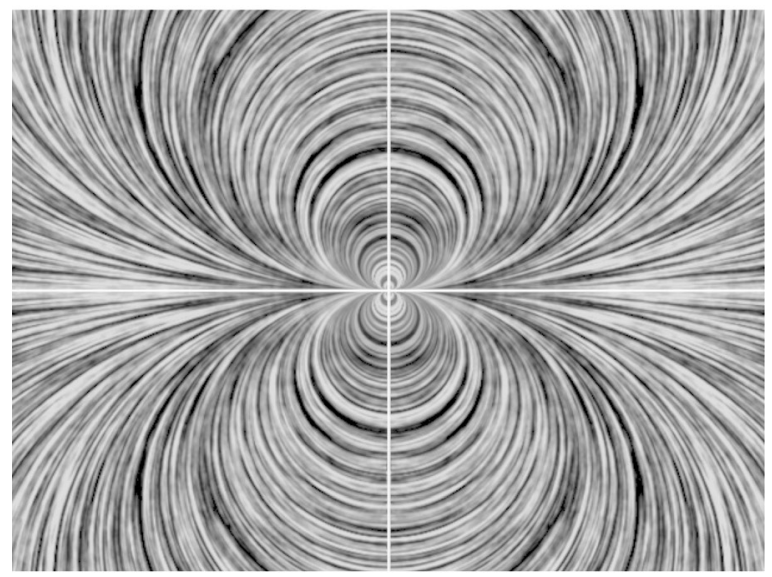

(b)

Fig. 4. Line integral convolutions showing the eigenvector fields for the vertically oriented static gravitational point quadrupole. The top image shows the field for negative eigenvalues, and the bottom image shows the field for positive eigenvalues.

$\mathbf{g}=-\nabla \Phi_{g}$, with components $\partial \Phi / \partial x^{j}$, but the tensorial quantity $\mathcal{E}$ with the components $\partial^{2} \Phi_{g} / \partial x^{j} \partial x^{k}$. The component notation correctly suggests that $\mathcal{E}$ is the gradient ${ }^{20}$ of $\mathbf{g}$. Why not, then, simply visualize gravitational fields with images of g? For that matter, why not simply use potentials in both cases?

In electromagnetism we know the answer. The electrostatic potential is useful for electrostatics, but the concept does not carry over to time-changing fields, and hence to radiation. For electromagnetic radiation, the electric field $\mathbf{E}$ is a valid and important concept, but it is not simply the gradient of a scalar. The same turns out to be true in gravitation. For gravitational radiation, $\mathcal{E}$ is a valid and important concept, but its components are not the second derivatives of a scalar field.

\section{DYNAMIC FIELDS}

\section{A. Electromagnetic fields}

\section{General visualization considerations}

While LIC snapshots are useful, they show the fields only at a moment in time and do not capture the necessarily dynamic nature of the radiation fields. To address this shortcoming, a new technique, dynamic line integral convolution (DLIC), has been developed. ${ }^{1,21}$ Quite aside from the 


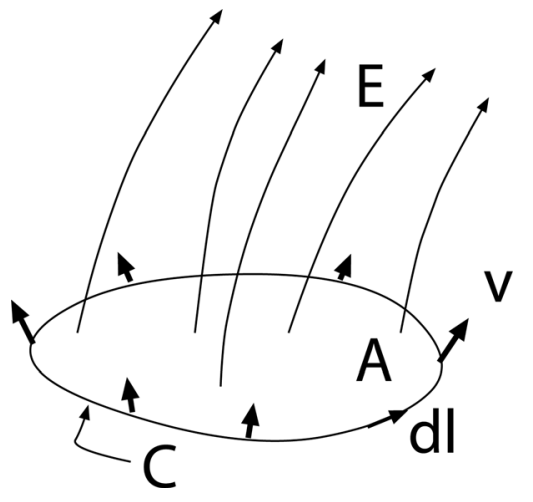

Fig. 5. Electric flux $\mathbf{E}$ through an area whose boundary is moving with velocity $\mathbf{v}$.

technical challenges to be overcome is a fundamental question underlying the notion of field line movement. Animations can show how a certain field line changes from one moment to the next, but what do we mean by "a certain field line"? How do we relate the field line at one moment to the "same" field line at another moment? How do we put unchanging "tags" on a field line? ${ }^{22}$ It turns out that there is a reasonable criterion for tagging field lines. At least in the case of axisymmetric sources, the mathematical nature of Maxwell's equations imposes these tags in a natural way. Furthermore, the same tagging can be justified on purely physical grounds.

The mathematical property is illustrated in Fig. 5. We consider an area $A$, with unit normal $\mathbf{n}$, bounded by curve $C$ and moving with velocity $\mathbf{v}$. The flux of electric field through $A$ then changes in time according to 23

$$
\begin{aligned}
\frac{d}{d t} \int_{A} \mathbf{E} \cdot \mathbf{n} d A & =\int_{A} \frac{\partial \mathbf{E}}{\partial t} \cdot \mathbf{n} d A+\int_{C}(\mathbf{E} \times \mathbf{v}) \cdot d \mathbf{l} \\
& =\int_{A} c^{2} \nabla \times \mathbf{B} \cdot \mathbf{n} d A+\int_{C}(\mathbf{E} \times \mathbf{v}) \cdot d \mathbf{l} \\
& =\int_{C}\left(c^{2} \mathbf{B}+\mathbf{E} \times \mathbf{v}\right) \cdot d \mathbf{l}
\end{aligned}
$$

We adopt flux conservation as our criterion for the motion of electric field lines. This requires that the field line velocity $\mathbf{v}$ satisfy

$$
c^{2} \mathbf{B}+\mathbf{E} \times \mathbf{v}=0 .
$$

For magnetic field lines, the equivalent condition is

$$
\mathbf{E}+\mathbf{v} \times \mathbf{B}=0 .
$$

The component of $\mathbf{v}$ along the field lines is meaningless, so these conditions give one constraint on the components of $\mathbf{v}$ perpendicular to the field lines. In the case of axisymmetry, to be considered below, that is all we need.

For a magnetic field configuration, e.g., an oscillating magnetic dipole, we can interpret Eq. (21) as the condition that a charged particle experience no net Lorentz force. Particles trapped in tight orbits around magnetic field lines $d o$, in fact, move with field lines, so this condition has a very practical meaning in many plasma situations: the motion of field lines is equivalent to the motion of electrons trapped on tight orbits around field lines. For an electric field these same considerations apply if we replace the electric monopole charge motion with the motion of a (hypothetical) magnetic monopole charge.

In the radiation field, and in many nonradiative configurations, the electric and magnetic fields are orthogonal $(\mathbf{E} \cdot \mathbf{B}=0)$. In this case, the solutions to Eqs. (20) and (21) are, respectively,

$$
\mathbf{v}=c^{2} \frac{\mathbf{E} \times \mathbf{B}}{E^{2}}, \quad \mathbf{v}=\frac{\mathbf{E} \times \mathbf{B}}{B^{2}} .
$$

The numerator in both cases is proportional to the Poynting flux, indicating that the flux-conserving motion of the field lines is compatible with the transport of electromagnetic energy.

\section{Oscillating electric point quadrupole}

We can convert the static quadrupole of Fig. 1 to a quadrupole source oscillating at frequency $\omega$ by replacing the static distances $d$ with the oscillating distances $d_{0}+\Delta d \cos \omega t$. The "amplitude" of the quadrupole then changes from $Q=q d^{2}$ to

$$
\begin{aligned}
Q(t) & =q\left(d_{0}+\Delta d \cos \omega t\right)^{2} \\
& =q d_{0}^{2}+2 q d_{0} \Delta d \cos \omega t+q(\Delta d \cos \omega t)^{2} .
\end{aligned}
$$

We now take only the part of this expression that oscillates at frequency $\omega$, and we define $Q \equiv 2 q d_{0} \Delta d$. With this meaning for $Q$, the spherical components of the fields produced by this source $\operatorname{are}^{24}$

$$
\begin{aligned}
& E_{r}=-2 \frac{Q k^{2}}{4 \pi \epsilon_{0} r^{2}}\left[\cos (k r-\omega t)\left(1-\frac{3}{k^{2} r^{2}}\right)-\frac{3}{k r} \sin (k r-\omega t)\right]\left(\frac{3}{2} \cos ^{2} \theta-\frac{1}{2}\right), \\
& E_{\theta}=-\frac{Q k^{2}}{4 \pi \epsilon_{0} r^{2}}\left[\sin (k r-\omega t)\left(k r-\frac{6}{k r}\right)+\left(3-\frac{6}{k^{2} r^{2}}\right) \cos (k r-\omega t)\right] \cos \theta \sin \theta, \\
& B_{\phi}=-\frac{Q k^{3}}{4 \pi \epsilon_{0} c r}\left[\left(1-\frac{1}{3 k^{2} r^{2}}\right) \sin (k r-\omega t)+\frac{3}{k r} \cos (k r-\omega t)\right] \cos \theta \sin \theta,
\end{aligned}
$$

where $k \equiv \omega / c$. 
In the limit $k r \rightarrow 0$, the dominant terms in the field are proportional to $1 / r^{4}$, and these terms agree precisely with those in Eqs. (14). This leads to an important insight about visualization-as $k r$ becomes much smaller than unity the dominant terms in the field are the $1 / r^{4}$ terms, and these terms have the form of the static solution modulated by $\cos \omega t$. In other words, at distances from the source much less than a wavelength, the field is a quasistatic field: a field with the structure of the static field, but with an amplitude oscillating in time.

Far from the source, in the region $k r \gg 1$, the story is very different. Here, the radial electric field falls off as $1 / r^{2}$. The dominant fields are the $1 / r$ parts of $E_{\theta}$ and $B_{\phi}$

$$
\begin{aligned}
& E_{\theta}=-\frac{Q k^{3}}{4 \pi \epsilon_{0} r} \sin (k r-\omega t) \cos \theta \sin \theta, \\
& B_{\phi}=-\frac{Q k^{3}}{4 \pi \epsilon_{0} c r} \sin (k r-\omega t) \cos \theta \sin \theta .
\end{aligned}
$$

These are the radiation fields, that is, the fields in the limit where $r$ is much larger than the radiation wavelength. The fact that the radial component does not contribute to this field is simply a statement that the radiation fields are transverse, orthogonal to the direction to the source.

Figure 6 shows an LIC snapshot of the fields of the oscillating electric quadrupole at a single instant of time. The wavelength $\lambda=c / \omega=c T$ of the radiation is indicated on the figure, and it is particularly interesting to note that the central region of Fig. 6 - the region small compared to $\lambda$-is indistinguishable from Fig. 2. In the opposite limit, far from the central region, the figure shows (to the extent that it includes far-away regions) that the field tends to take the radiation form, with transverse field lines. What is most interesting in Fig. 6, however, is the intermediate region. The figure reveals details of the structure of the transition fields ${ }^{1}$ that cannot easily be inferred from the mathematical expressions in Eqs. (24) and (25).

A snapshot of a dynamic field, however, necessarily shows only a single phase of the radiation field. A full description requires an animation, and an animation requires a way of identifying field lines at different moments of time, as described in Sec. III A. The identification principle gives a

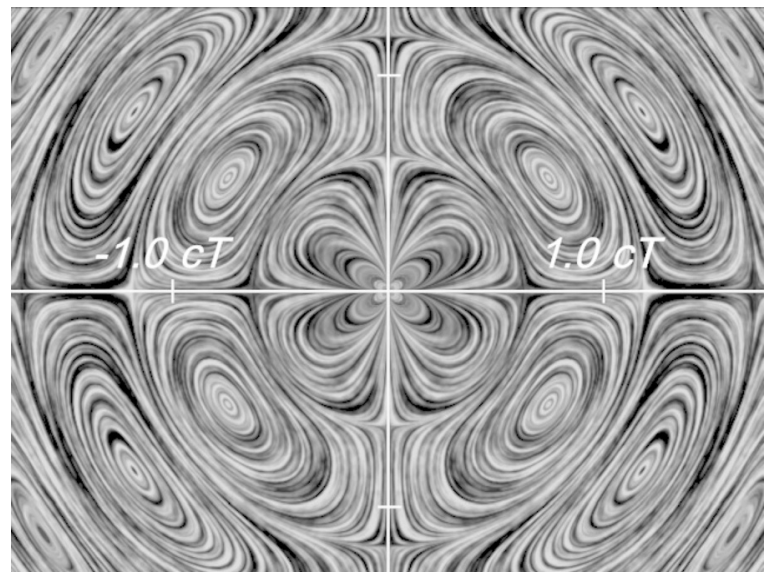

Fig. 6. A line integral convolution snapshot of the field of an oscillating electric quadrupole [Eqs. (24) and (25), with $t=0$ ]. The markers show points that are one wavelength $(c T)$ from the origin. At distances from the origin small compared to one wavelength, the solutions approach those of the static solutions shown in Fig. 2. A movie of this radiating quadrupole is available online and as supplementary material ${ }^{25}$ (enhanced online) [URL: http:// dx.doi.org/10.1119/1.4807853.1].

single constraint on the velocity of field lines orthogonal to the line itself. In the axisymmetric case, geometry gives us the other constraint. In particular, the electric field lines in Fig. 1, by symmetry, can have no $\phi$ component. The condition in Eq. (20) then completely fixes the line velocity v. A DLIC animation of the dynamic electric field lines is linked to the online version of Fig. 6, and this and several related videos are available in the online supplement. ${ }^{25}$

\section{B. Gravitational fields}

\section{Oscillating gravitational point quadrupole}

To create a point quadrupole gravitational source oscillating at frequency $\omega$, we can perform precisely the same procedure as for the electromagnetic case. We take the distance $d$ to have the form $d_{0}+\Delta d \cos \omega t$ and we take the symbol $Q$ to mean $2 M d_{0} \Delta d$. The nonvanishing spherical components of $\mathcal{E}$ and $\mathcal{B}$, the analogs of Eqs. (24)-(26), are found to be

$$
\begin{aligned}
& \mathcal{E}_{r r}=\frac{-4 G Q k^{2}}{r^{3}}\left[\left(-1+\frac{3}{k^{2} r^{2}}\right) \cos (k r-\omega t)+\frac{3 \sin (k r-\omega t)}{k r}\right]\left(3 \cos ^{2} \theta-1\right), \\
& \mathcal{E}_{r \theta}=\frac{-4 G Q k^{2}}{r^{3}}\left[\left(\frac{6}{(k r)^{2}}-3\right) \cos (k r-\omega t)-\left(k r-\frac{6}{k r}\right) \sin (k r-\omega t)\right] \sin \theta \cos \theta, \quad(30) \\
& \mathcal{B}_{r \phi}=\frac{-4 G Q k^{2}}{c r^{3}}\left[-3 \cos (k r-\omega t)-\left(k r-\frac{3}{k r}\right) \sin (k r-\omega t)\right] \sin \theta \cos \theta, \\
& \mathcal{E}_{\phi \phi}-\mathcal{E}_{\theta \theta}=\frac{-2 G Q k^{2}}{r^{3}}\left[\left(-\frac{3}{k^{2} r^{2}}+3-(k r)^{2}\right) \cos (k r-\omega t)-\left(\frac{3}{k r}-2 k r\right) \sin (k r-\omega t)\right] \sin ^{2} \theta,
\end{aligned}
$$




$$
\mathcal{B}_{\theta \phi}=\frac{-G Q k^{2}}{c r^{3}}\left[\left(-3+k^{2} r^{2}\right) \cos (k r-\omega t)-\left(-\frac{3}{k r}+2 k r\right) \sin (k r-\omega t)\right] \sin ^{2} \theta .
$$

As was the case for the electromagnetic fields in Eqs. (24) through (26), there are interesting limits to these expressions for both $k r \ll 1$ and $k r \gg 1$. In the former case, we find that to the leading $1 / r^{5}$ order, $\mathcal{E}_{r r}, \mathcal{E}_{r \theta}$, and $\mathcal{E}_{\phi \phi}-\mathcal{E}_{\theta \theta}$ have the form of Eqs. (18), modulated by $\cos \omega t$, while the gravitomagnetic components vanish to this order. As in electromagnetism, at distances from the source much less than a wavelength, the fields are those of a quasistatic source; that is, they have the structure of a static field but oscillate in time.

In the opposite limit $(k r \gg 1)$, the dominant components fall off as $1 / r$

$$
\begin{aligned}
& \mathcal{E}_{\phi \phi}=-\mathcal{E}_{\theta \theta}=\frac{G Q \omega^{4}}{r} \cos (k r-\omega t) \sin ^{2} \theta, \\
& \mathcal{B}_{\theta \phi}=-\frac{G Q \omega^{4}}{r} \cos (k r-\omega t) \sin ^{2} \theta .
\end{aligned}
$$

The form of these radiation fields suggests that they are transverse, as can be confirmed with the mathematics of general relativity and an appropriate set of definitions and constraints. For our purposes, here, it is sufficient to note that the eigenvector directions can immediately be inferred. From Eq. (34), and treating $\mathcal{E}_{r r}$ as zero, we have that the matrix of gravitoelectric field components is diagonal and hence that there are eigenvectors of the gravitoelectric field in the $\theta$-direction, in the $\phi$-direction, and (with zero eigenvalue) in the $r$-direction. From Eq. (35) we infer that in the radiation zone there are two eigenvectors for the gravitomagnetic field in the $\theta \phi$-plane, each at $45^{\circ}$ from the $\theta$ and the $\phi$ directions. Again there is an eigenvector field, with zero eigenvalue, in the radial direction.

Snapshots of the eigenlines of $\mathcal{E}$ are shown in Fig. 7. As in the electromagnetic case, these snapshots show what we already learned from the mathematics. Close to the source the eigenline field is quasistatic, and far from the source it is transverse. Also as in the electromagnetic case, these figures can clarify what the mathematics cannot-the form of the fields in the intermediate zone, at distances from the source comparable to the wavelength $\lambda=c T$. In this zone the fields must make a transition from the quasistatic small- $r$ form to the radiating large- $r$ form.

Both in the mathematics and in Fig. 7, we see that the form of the fields in the intermediate zone is even richer for the oscillating gravitational quadrupole than for the oscillating electric quadrupole. In the latter case, the radiation fields fall off as $1 / r$, while the near-source quasistatic field is proportional to $1 / r^{4}$. For the gravitational quadrupole, the radiation fields have the same $1 / r$ character, but the near-source quasistatic fields are proportional to $1 / r^{5}$.

There is an interesting feature of the gravitational fields that is associated with the mathematical expressions more than the visualizations. The expressions in Eqs. (24)-(26), for $E_{r}, E_{\theta}$, and $B_{\phi}$, are identical to those for $\mathcal{E}_{r r}, \mathcal{E}_{r \theta}$, and $\mathcal{B}_{r \phi}$ when the change $1 / 4 \pi \epsilon_{0} r^{2} \rightarrow-2 G Q / r^{3}$ is made; this is true not only in the near-source zone or the radiation zone, but for all values of $k r$ ! This means that, aside from a change in constants and a single factor of $r$, the electromagnetic solution, including radiation fields, is completely contained in the nonradiative part of the gravitational solution. Stated in the other direction, the electromagnetic solution contains all of the gravitational solution except for the components $\mathcal{E}_{\phi \phi}-\mathcal{E}_{\theta \theta}$ and $\mathcal{B}_{\theta \phi}$, which carry radiation [cf. Eqs. (34) and (35)].

This correspondence, of course, is not a coincidence, nor is it an idiosyncrasy of the axially symmetric point quadrupole. Rather it is a consequence of the structure of classical theories that describe massless fields (fields that propagate at the speed of light). This structure is most apparent when the fields are described with the appropriate mathematics: a set of scalars that result from projecting the fields onto a set of basis vectors best suited to the analysis of propagating fields. ${ }^{27,28}$

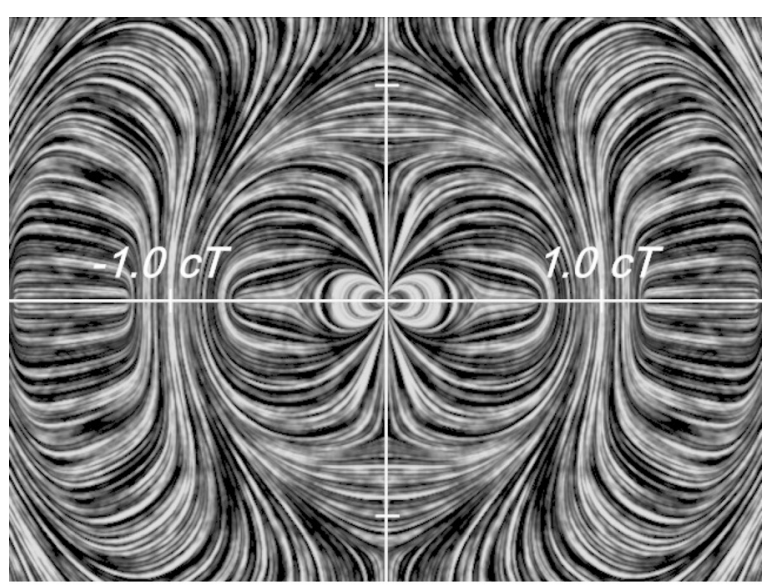

(a)

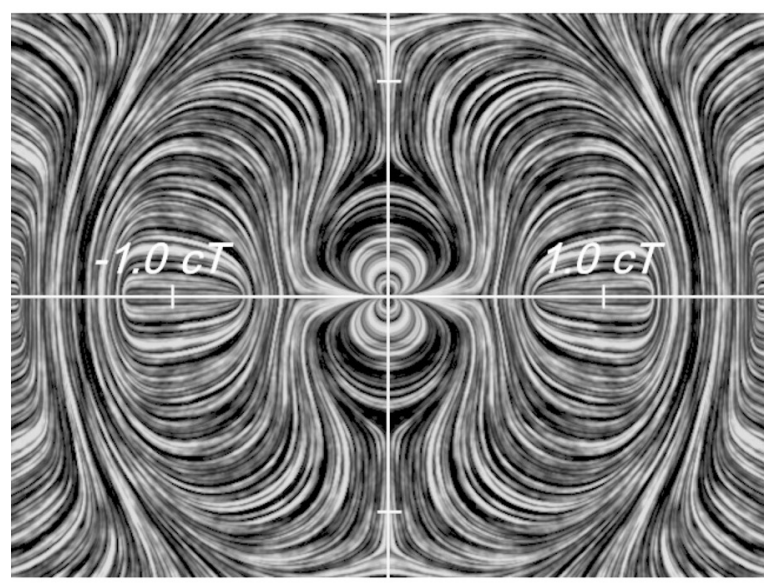

(b)

Fig. 7. Line integral convolution snapshots of the eigenlines of the gravitoelectric field $\mathcal{E}$ of an oscillating point gravitational quadrupole described by Eqs. (29), (30), and (32) at time $t=0$. The markers show points that are one wavelength $(c T)$ from the origin. The top image shows the eigenlines for one family ${ }^{26}$ of eigenvectors; the bottom image shows the other family. At distances from the origin small compared to the wavelength, the fields, and hence the eigenlines, approach those of the two families of eigenlines for the static solution shown in Fig. 4. Movies for this radiating quadrupole are available online and as supplementary material ${ }^{25}$ (enhanced online) [URL: http://dx.doi.org/10.1119/1.4807853.2] [URL: http://dx.doi.org/10.1119/ 1.4807853.3]. 
This relationship of the electromagnetic and linearized gravity fields gives us an additional possibility for visualization. If we project the gravitational tensors with the unit $r$ vector (equivalently, take the dot product of $\mathcal{E}$ and $\mathcal{B}$ with the unit radial vector $\hat{\boldsymbol{r}}$ ), we get two vector fields, one with $r, \theta, \phi$ components $\mathcal{E}_{r r}, \mathcal{E}_{r \theta}, \mathcal{E}_{r \phi}$, the other with components $\mathcal{B}_{r r}, \mathcal{B}_{r \theta}, \mathcal{B}_{r \phi}$. The $\mathbf{E}$ field differs from the vector $\left(\mathcal{E}_{r r}, \mathcal{E}_{r \theta}, \mathcal{E}_{r \phi}\right)$ only by a factor of $r$, and hence the direction of the two fields is the same at any point. The same is true for the corresponding $\mathbf{B}$ field. The LICs of $\mathbf{E}$ and $\mathbf{B}$, as in Fig. 6, can therefore be considered to be LICs of all parts of the gravitational field except those transverse to the $r$ direction. It is those transverse parts, of course, that carry the radiation.

\section{General visualization considerations}

As in the electromagnetic case, we have provided videos (either linked to the online article or available as supplementary material ${ }^{25}$ ) to show the time evolution of the gravitational radiation fields in Fig. 7. However, in linearized gravity, there is no equivalent of the principles that define and constrain field line motion in electromagnetism. This means that we can show a sequence of eigenlines, but we cannot say which line at one moment corresponds to which line at another. This fundamental problem turns out not to be a barrier if we only want to show the qualitative nature of the field pattern motions in gravitation. Intuitively useful flow fields can be guessed at qualitatively due to the existence of singularities.

In plots of lines tangent to vector fields, the singularities are points at which the vector has zero magnitude, so that the direction of a tangent line is undefined. In Fig. 6, such points can be seen both in the equatorial plane and along the symmetry axis. If the vector field illustrated were the velocity of a fluid, these singular points would be called stagnation points.

It is simple to argue that lines of the electric field $\mathbf{E}$ in electromagnetic quadrupole radiation moving according to the rules of Eq. (20) do not cross singularities. Similarly, in the gravitational case singularities are easily identified and the motion of the singularities - their displacement from one snapshot to another-therefore gives us a coarse visual sketch of the motion of the field lines; once the position of the singularities is known at a new time, the remainder of the field lines can be approximately drawn in. Different choices of the details of how they are drawn make no difference in the qualitative content of the images.

Note that the eigenline fields of gravitational radiation shown in Fig. 7 are richer in singularities than the electromagnetic vector fields shown in Fig. 6. In addition to "stagnation points," the eigenlines contain line singularities in the equatorial plane. In sequences of LIC snapshots, the motion of these singularities, and the understanding that they "drag" the field lines, give approximate meaning to the evolution of the eigenlines. Such qualitative visual flow fields could be used in making the movies such as those that accompany this article. However, we chose not to do that even qualitatively and have taken the underlying flow field to be zero in those movies. The eye of the observer provides a concept of motion in any case, and we felt that it would be overinterpretation to try to augment that perception by imposing a qualitative flow pattern that matches it.

It is interesting, in any case, that there is no "correct" or "true" motion of the eigenlines. There is no physical process to define the motion of the lines, analogous to the spiraling of charged particles around magnetic field lines or the identification of specific fluid elements in velocity flows. Nor does any mathematical criterion, such as flux conservation, constrain the motion of field lines. Exploiting the locations of singularities is the best we can do.

The intuitive feeling that there should be a well-defined meaning to the motion of field lines may be rooted in the relationship of electromagnetic field line motion and energy flow - a relationship, expressed in Eq. (22), that is limited to regions, such as the radiation region, in which the $\mathbf{E}$ and $\mathbf{B}$ fields are orthogonal. This relationship raises the question of whether energy flow could be used as a guide to the motion of eigenlines, and hence whether there is a gravitational ana$\log$ of the Poynting flux.

There is, in fact, a pragmatic quantitative measure of energy flow in gravitational waves: the Landau-Lifschitz pseudotensor. ${ }^{29}$ But this measure is neither definitive nor useful for our purposes. The fact that it is not definitive is important. Just as gravitational acceleration near the Earth's surface vanishes in a freely falling elevator, many other aspects of gravitation vanish in an appropriately chosen reference frame. As a consequence, it is fundamentally impossible to localize energy in a gravitational wave. The Landau-Lifschitz pseudotensor can give only a sort of average energy flux over several wavelengths. Such a measure is not useful for us because it cannot help us give meaning to line motion. The mathematical details confirm this suggestion-the pseudotensor cannot be inferred from the gravitoelectric and gravitomagnetic fields. In a very rough sense, the pseuodensor is constructed from mathematical objects that are spacetime integrals of $\mathcal{E}$ and $\mathcal{B}{ }^{30}$

\section{CONCLUSIONS}

We have compared the mathematics and visualizations of electromagnetic and gravitational fields, working up from static field configurations to radiating oscillatory versions of these configurations, focusing on the examples of electric and gravitational point quadrupoles. Visualizations, using line integral convolutions (LICs), were based on the familiar field lines for the electromagnetic case and on the tendex/ vortex eigenline formalism ${ }^{2-4}$ in the gravitational case.

We have found, as foretold in the Introduction, that this comparison shows both instructive similarities and instructive differences. An important similarity in the radiation examples is the transition, in both cases, from a quasistatic field structure at distances from the point source small compared to a wavelength, to a transverse, $1 / r$ radiation field at distances large compared to a wavelength. The visualizations, in both cases, show field structures that are not easily seen in the mathematics, and show how the fields make the transition from the near-source structure to the very different radiation structure.

An important difference between the two cases lies in the visualization of dynamical fields. The motion of electromagnetic field lines has both mathematical and physical meaning, while the motion of gravitational eigenlines has neither. This difference can be partially ascribed to the difference between the nature of eigenlines for a tensor field and "lines of force" for a vector field. But the difference, especially regarding energy flow, underscores fundamental differences between electromagnetism and relativistic gravitation.

In Sec. IV B, there was a rediscovery and illustration of an interesting mathematical relationship between the 
electromagnetic and gravitational fields. Aside from trivial replacements, the complete structure of the electromagnetic solution-including the radiation fields-is contained within the gravitational field solution. To go from the electromagnetic solution to the gravitational requires only adding the gravitational radiation fields.

\section{ACKNOWLEDGMENTS}

For discussions of tendex/vortex lines, the authors thank the groups at Caltech and Cornell, in particular, Kip Thorne, Mark Scheel, Rob Owen, Jeff Kaplan, Fan Zhang, and Aaron Zimmerman.

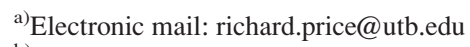

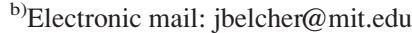

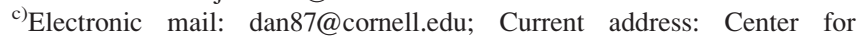
Radiophysics and Space Research, Cornell University, Ithaca, New York 14853.

${ }^{1}$ J. Belcher and C. Koleci, "Using animated textures to visualize electromagnetic fields and energy flow," e-print arXiv:0802.4034.

${ }^{2}$ R. Owen et al., "Frame-dragging vortexes and tidal tendexes attached to colliding black holes: Visualizing the curvature of spacetime," Phys. Rev. Lett. 106, 151101 (2011).

${ }^{3}$ D. A. Nichols et al., "Visualizing spacetime curvature via frame-drag vortexes and tidal tendexes: General theory and weak-gravity applications," Phys. Rev. D 84, 124014 (2011).

${ }^{4}$ A. Zimmerman, D. A. Nichols, and F. Zhang, "Classifying the isolated zeros of asymptotic gravitational radiation by tendex and vortex lines," Phys. Rev. D 84, 044037 (2011).

${ }^{5} \mathrm{We}$ omit special relativistic effects here, such as the Lorentz factor. We assume that motions are slow compared to the speed of light.

${ }^{6}$ C. W. Misner, K. S. Thorne, and J. A. Wheeler, Gravitation (W. H. Freeman and Co., San Francisco, 1973), Sec. 36.1.

${ }^{7}$ K. S. Thorne, R. H. Price, and D. A. MacDonald, Black Holes: The Membrane Paradigm (Yale U.P., New Haven, 1986).

${ }^{8}$ In fully nonlinear general relativity the gravitoelectric and gravitomagnetic tensors are defined as projections of the Weyl tensor (equal to the Riemann tensor in source-free spacetime). For details see Ref. 3.

${ }^{9}$ See Ref. 7, Eq. (5.21).

${ }^{10}$ In general relativity these equations follow from the "Bianchi identities," mathematical identities satisfied by the Riemann tensor. Since $\mathcal{B}$ has no Newtonian equivalent these equations cannot be understood from simple Newtonian gravity theory.

${ }^{11}$ The literature on general relativity often adopts units in which $c=1$. As pointed out in Sec. I, we do not make that choice here. In accordance with Eq. (8), with our definitions $\mathcal{E}$ has dimensionality $1 /$ time $^{2}$ and $\mathcal{B}$ has dimensions $1 /$ (time $\cdot$ length).

${ }^{12} \mathrm{We}$ emphasize this point because weak relativistic gravitational fields are often described with metric perturbations $h_{\mu \nu}$. These perturbations, like electromagnetic potentials, are mathematically useful, but they are subject to gauge transformations and hence do not directly represent physical effects.

${ }^{13}$ B. Cabral and C. L. Leedom, "Imaging vector fields using line integral convolution," in Proceedings of the SIGGRAPH 93, edited by J. T. Katiya (ACM Press, 1993), pp. 263-270.

${ }^{14}$ J. D. Jackson, Classical Electrodynamics, 3rd ed. (Wiley, Hoboken, 1998).

${ }^{15}$ This ellipsoid can be drawn only if the three principal moments of inertia are all positive, but the fact that mass density is nonnegative does guarantee this. The ellipsoid cannot be used so simply for, say, $\mathcal{E}_{j k}$ because its tracelessness means that the sum of its principal values is zero.
${ }^{16}$ T. Delmarcelle and L. Hesselink, "The topology of symmetric, secondorder tensor fields," in Proceedings of the Conference on Visualization 94, edited by D. Bergeron and A. Kaufman (IEEE Computer Society Press, Los Alamitos, CA, 1994), pp. 140-147.

${ }^{17}$ This inclusion is made for simplicity to eliminate the mass monopole when the $d \rightarrow 0$ limit is taken. With the $-2 M$ particle omitted, the argument would require eliminating the monopole moment by other means, such as a specific projection of only the quadrupole moment.

${ }^{18}$ Although the static components are straightforward to compute with Eq. (6), this procedure is valid only for Cartesian components. To arrive at useful results those components must subsequently be transformed to the spherical basis and to functions of $r$ and $\theta$. In practice, it is much simpler to use the formalism of tensorially correct differentiation ("covariant differentiation"); see J. Romano and R. H. Price, "Why no shear in 'Div, grad, curl, and all that'?," Am. J. Phys. 80, 519-524 (2012).

${ }^{19}$ In the gravitational case this follows from the fundamental definition of $\mathcal{B}$ in general relativity. The tensor $\mathcal{B}$ involves the components of a fourth rank curvature tensor with only a single time (i.e., 0) index. Such components reverse sign under time reversal. In static configurations these components must be unchanged under time reversal, hence they must be zero. The vanishing of $\mathcal{B}$ for static configurations is also suggested by Eq. (10).

${ }^{20}$ Due to the choice of sign in Eq. (6), $\mathcal{E}$ is actually the negative of the gradient of $\mathbf{g}$.

${ }^{21}$ A. Sundquist, "Dynamic line integral convolution for visualizing streamline evolution," IEEE Trans. Vis. Comput. Graph. 9(3), 273-282 (2003).

${ }^{22}$ This issue is discussed in detail in J. W. Belcher and S. Olbert, "Field line motion in classical electromagnetism," Am. J. Phys. 71, 220-228 (2003).

${ }^{23}$ The second term on the right-hand-side of the first line accounts for the change in flux due to the motion of the curve $C$ bounding the area A. The mathematics here for time changing electric flux is identical to that for the time changing magnetic flux in Faraday's law. See, e.g., Eq. (5.137) and the associated footnote in Ref. 14.

${ }^{24}$ These fields are most simply computed using the vector spherical harmonics in Sec. 9.10 of Ref. 14.

${ }^{25}$ See supplementary material at http://dx.doi.org/10.1119/1.4807853 for a variety of related videos in addition to the videos linked to the online versions of Figs. 6 and 7; see also http://web.mit.edu/viz/gravrad/.

${ }^{26}$ Each curve in Fig. 7 corresponds to a particular eigenvalue. Each family represents a set of eigenvalues and of eigenvector components that vary smoothly from one curve to the adjacent curve. It turns out that the signs of the eigenvalues vary within a family. The eigenvalues for the top LIC in Fig. 7 are negative in both the near zone and the radiation zone, but positive in a part of the intermediate zone. The opposite applies to the bottom LIC.

${ }^{27}$ E. Newman and R. Penrose, "An approach to gravitational radiation by a method of spin coefficients," J. Math. Phys. 3, 566-578 (1962). In such a formalism all field theories consist of the same equations relating the scalars, and differ only in the number of scalars. There are three such (complex) scalars for electromagnetism describing the six components of $\mathbf{E}$ and B. There are five such (complex) scalars for the ten independent components of $\mathcal{E}$ and $\mathcal{B}$. The two extra scalars are linear combinations of the components $\mathcal{E}_{\phi \phi}-\mathcal{E}_{\theta \theta}, \mathcal{B}_{\theta \phi}, \mathcal{B}_{\phi \phi}-\mathcal{B}_{\theta \theta}$, and $\mathcal{E}_{\theta \phi}$ that carry information about radiation. (The latter two components vanish for our quadrupole example.) If the field equations that contain these extra two scalars are eliminated, then the field equations for the gravitational scalars - multiplied by $r$-are the same as those of electromagnetism.

${ }^{28}$ R. H. Price, "Nonspherical perturbations of relativistic gravitational collapse. II. Integer-spin, zero-rest-mass fields," Phys. Rev. D 5, 2439-2454 (1972).

${ }^{29}$ See Sec. 35.15 of Ref. 6.

${ }^{30}$ The Landau-Lifschitz pseudotensor is constructed from the spacetime gradients of metric perturbations. The gravitoelectric and gravitomagnetic fields are constructed from the gradients of the gradients of the metric perturbations. 